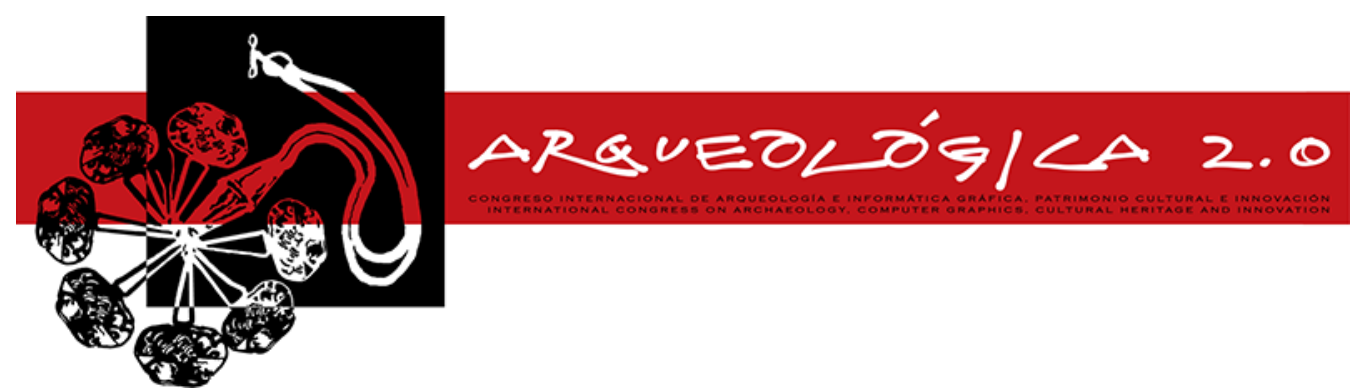

Proceedings of the $8^{\text {th }}$ International Congress

on Archaeology,

Computer Graphics,

Cultural Heritage and Innovation

'ARQUEOLÓGICA 2.0'

in Valencia (Spain),

Sept. 5-7, 2016

DOI: http://dx.doi.org/10.4995/arqueologica8.2016.3564

Received: 07/03/2016

Accepted: 04/05/2016

\title{
SIMULACIÓN 3D CON INTERFACES HÁPTICAS PARA LA RECUPERACION DEL PATRIMONIO CULTURAL DEL TREN EN RIOBAMBA
}

\author{
3D HAPTIC INTERFACES SIMULATION FOR RECOVERY OF CULTURAL HERITAGE TRAIN IN RIOBAMBA \\ Miguel Duque *, Fernando Proaño, Ramiro Santos \\ Grupo de Investigación 3D-FIE-ESPOCH, Escuela Superior Politécnica de Chimborazo, Panamericana Sur Km 1 1⁄2, EC060155, \\ Riobamba, Ecuador. m duque@espoch.edu.ec; fproanio@espoch.edu.ec; ramiro.santos2005@gmail.com
}

\begin{abstract}
:
The present work had studied and determined various levels of usability and interactivity in haptic interfaces for manipulating 3D applications. It was applied to a Heritage Cultural system composed by Train Station and Steam Locomotive. A physical model and a railroad model were built. The system was operated by high school students. The information gathering was performed by the deductive method, collecting data about preferences, needs, and requirements for developing a 3D virtual environment using 3DSMAX 2011 Trial (3D Modeled Software) and Unity 4.3 Trial (Simulation Software), capable to engage and transmit information relevant to geographical, social and cultural aspects of the central highlands. Subsequently connected to a real model controlled by electronic systems. The inquiry made from high school students shows that $56 \%$ possess ability and willingness for interfaces manipulation. Also, it was found an $80 \%$ of criteria for usability acceptance, and interactivity of $84 \%$ towards the current applications on mobile devices. As the final evaluation of the application, it was shown that the intervention of haptic interfaces on threedimensional applications connected to tangible environments, increased $95 \%$ of the usability and $100 \%$ of interactivity. We conclude that combining virtual systems with real ones accelerates learning processes at the secondary education level. It improves retention of information and allows the user to actively participate in virtual environments, converting recreational applications into academic and research tools.
\end{abstract}

Key words: haptic interface, interactivity, cultural heritage

\section{Resumen:}

El presente trabajo estudió y determino diferentes El presente trabajo estudió y determino diferentes niveles de usabilidad e interactividad en interfaces hápticas para manipulación de aplicaciones 3D. Se aplicó a un Patrimonio Cultural que comprende la Estación del tren y una locomotora a vapor, los mismos que fueron representados en una maqueta física y un sistema de trenes eléctricos respectivamente. Este sistema fue operado por estudiantes secundarios. La obtención de información se realizó mediante el método deductivo, recolectando datos sobre necesidades y requerimientos para elaborar un entorno virtual 3D utilizando 3DSMAX 2011 Trial (Software Modelado 3D) y Unity 4.3 Trial (Software Simulación), capaz de vincularse y transmitir información relevante sobre aspectos geográficos, sociales y culturales de la serranía central. Posteriormente conectada a una maqueta real controlada por sistemas electrónicos. La indagación realizada a estudiantes, muestra que el $56 \%$ poseen capacidad y disposición de manipulación de interfaces, también se encontró aceptación del $80 \%$ en criterios de usabilidad y $84 \%$ en interactividad hacia aplicaciones actuales en dispositivos móviles, como evaluación final a la aplicación se demostró que la intervención de interfaces hápticas en aplicaciones tridimensionales conectadas a entornos tangibles, incrementaron la usabilidad al $95 \%$ y la interactividad al $100 \%$. Se concluye que combinar sistemas virtuales con reales acelera procesos de aprendizaje a nivel de educación secundaria, mejora la retención de información y permite al usuario participar activamente con entornos virtuales, convirtiendo aplicaciones lúdicas en herramientas académicas e investigativas.

Palabras clave: interfaces hápticas, interactividad, patrimonio cultural

"Corresponding Author: Miguel Duque, $\underline{m \text { duque@espoch.edu.ec }}$ 


\section{Introducción}

En la actualidad todo sistema que va a ser controlado por el hombre debe cumplir con aspectos técnicos de diseño centrado en el usuario y usabilidad que permitan obtener la mejor experiencia cuando el usuario maneja la aplicación, la importancia de una interfaz sencilla, amigable y usable determinan muchas veces que el usuario final se quede o abandone una aplicación.

En la actualidad el auge que los dispositivos móviles tienen entre los adolescentes ha provocado una serie de estudios sobre los aspectos positivos y negativos de los mismos, según un estudio independiente publicado en el portal Kubernética, a lo largo de las últimas décadas las nuevas tecnologías de la comunicación han tenido una enorme penetración en la sociedad y principalmente en los jóvenes, si bien este es un aspecto destacable de esta tecnología, pueden ser consideradas un riesgo si se vuelen adictivas y distractoras de actividades académicas como manifiesta un grupo de investigadores de la Universidad Autónoma del Estado de México (Méndez et al. 2015), sin embargo su facilidad de uso y el poco conocimiento necesario para instalar y configurar aplicaciones móviles han sido consideradas para que en el presente estudio sea considerado el dispositivo ideal sobre el cual funcionará la aplicación 3D que permita concientizar y revivir un patrimonio cultural de la ciudad de Riobamba entre los estudiantes de nivel secundario de esta ciudad.

Considerando las ventajas que los dispositivos móviles pueden brindar al desarrollo del proyecto se investigó la posibilidad de realizar interfaces hápticas (táctiles) utilizando software 3D de base y aplicaciones complementarias en las cuales se consideraron los principios y procesos de diseño gráfico, al final, se pudo combinar el uso de sistemas interactivos 3D en el modo digital y virtual con una integración innovadora a sistemas físicos analógicos de una maqueta con elementos de transporte como es el ferrocarril ecuatoriano. La aplicación final 3D entró en una etapa de análisis, para medir los niveles de interactividad y usabilidad de los usuarios en una aplicación interactiva eligiendo a la estación del tren de Riobamba, considerado un Patrimonio Cultura de la ciudad. De igual manera se analizó e identificó el nivel de usabilidad e interactividad que puede alcanzar una interfaz háptica al momento de manipular una aplicación y a su vez identificar parámetros técnicos que debe poseer una interfaz háptica para programarla en un sistema interactivo $3 \mathrm{D}$ y posteriormente integrarla a una maqueta física real (Aparici y Silva 2012).

El procedimiento de investigación utilizado fue el método deductivo, debido a que se hizo una revisión sistemática de los casos generales existentes y documentados hasta la actualidad y aplicarlos en un caso particular formulado para el presente estudio.

\section{Marco teórico}

Considerando que el diccionario de la lengua española define la palabra háptico/a como táctil, la presente investigación pretende utilizar esta característica para crear una interfaz que sea probada a través de dispositivos móviles (smartphone, tablets) para controlar el ambiente simulado del recorrido del tren de la ciudad de Riobamba, aquí nace una importante pregunta, ¿qué son las interfaces hápticas? los autores del trabajo Interfaz háptica para tareas de manipulación las describen como aquellas interfaces que permiten al usuario interactuar con sistemas tele operados en entornos remotos o con objetos simulados en entornos virtuales (López et al. 2010), por su parte el portal EcuRed (2016) amplía el termino y habla de tecnologías hápticas como el conjunto de interfaces tecnológicas que interaccionan con el ser humano mediante el sentido del tacto y que conjuntamente con nuevos métodos para generar contenido en realidad virtual científicos europeos ya han logrado teletransportar de forma virtual objetos reales a través del ciberespacio, algunas de las aplicaciones de esta tecnología puede ser observada en los simuladores hápticos empleados en entrenamiento de operaciones médicas y en simuladores de vuelo para el entrenamiento de pilotos, otro ejemplo de su actual uso, son los juegos, con las denominadas "tabletas vibradoras" ("rumble packs") con las que el usuario siente como hay irregularidades tales como un terreno desigual cuando conduce un automóvil, en medicina existen diversas interfaces hápticas en el caso de simulación médica y que pueden resultar útiles a la hora de intentar minimizar los daños ocasionados por el uso de procedimientos invasivos, a futuro esta tecnología podría utilizarse también para aplicaciones más profesionales que, por ejemplo, permitieran a médicos tratar pacientes a distancia o a diseñadores industriales colaborar de forma remota mediante la teletransportación a través de Internet de maquetas digitales palpables de sus diseños, otro ejemplo es el poder controlar un vehículo creado utilizando el kit Lego Mindstorms a través de una interfaz háptica generando un modelo de comunicación bidireccional mediante tecnología Bluetooth (Carrasquilla 2012). Sin duda el futuro permitirá acoplar esta tecnología a las actividades diarias y como manifiesta Andreas Schweinberger, coordinador de Immersence. "Los aspectos audiovisuales de la realidad virtual han avanzado mucho en los últimos años, por lo que añadir el sentido del tacto es un paso lógico".

La usabilidad es aplicada en cualquier caso donde exista una interacción entre un humano y un dispositivo, permitiendo al humano manipular, controlar y aprovechar de manera eficiente tanto el software como el hardware. El grado de usabilidad que se puede encontrar en la interacción humano-ordenador se basa en parámetros cuantificables permitiendo el establecimiento de niveles de usabilidad. La utilización de los gestos propios del usuario para interactuar con un sistema de comunicación puede resultar natural, directa e intuitiva (Cabral et al. 2005). Sin embargo, algunos autores también critican la naturalidad y la facilidad de intuición de estas interfaces y su usabilidad (Nielsen et al. 2003). Estos denominados niveles de usabilidad se los puede organizar con criterios de errores y opiniones técnicas (Tabla 1).

Coincidiendo en que la usabilidad es reconocida como un factor de calidad importante para sistemas interactivos incluidas las interfaces de usuario de una gran variedad de dispositivos y que el objeto de la evaluación de usabilidad de interfaces es utilizar los resultados para realizar una retroalimentación, con el fin de mejorar los diseños y por ende la interactividad con el usuario (Hurtado y Forero 2014), se realizó un test a estudiantes secundarios que en su mayoría utilizan 
dispositivos de pantalla táctil, siendo el celular el más utilizado seguido por las tabletas, cada criterio de usabilidad fue definido bajo tres funciones de pertenencia que son baja, media y alta como indica el sistema difuso basado en las reglas de Takagi-Sugeno, una vez tabulados los resultados, se obtuvo que alrededor del $80 \%$ de estudiantes consideran que las aplicaciones creadas en la actualidad son fáciles de usar, en un $56 \%$, los estudiantes muestran disposición y capacidad de manipular una interfaz con éxito, existiendo errores leves al momento de realizar tareas, y un $41,25 \%$ de los estudiantes encuestados consideraron que la aplicación sometida a la prueba, tienen un nivel de usabilidad medio, ubicándolo en la tercera posición de una escala del 1 al 5.

Tabla 1: Niveles de usabilidad

\begin{tabular}{|c|c|c|}
\hline Nivel & Grado de error & Descripción \\
\hline Nivel 5 & Fatal & $\begin{array}{l}\text { Error de concepto, errores que } \\
\text { impiden la realización de la tarea }\end{array}$ \\
\hline Nivel 4 & Grave & $\begin{array}{c}\text { Errores que confunden al usuario } \\
\text { disminuyendo la facilidad en el } \\
\text { ejercicio establecido }\end{array}$ \\
\hline Nivel 3 & Leve & $\begin{array}{l}\text { Problemas que pueden confundir } \\
\text { al usuario pero no afectan a la } \\
\text { conclusión de la tarea }\end{array}$ \\
\hline Nivel 2 & Cosmético & $\begin{array}{l}\text { Opiniones que no afectan el } \\
\text { desempeño de la aplicación, pero } \\
\text { afectan a su estética }\end{array}$ \\
\hline Nivel 1 & Nulo & $\begin{array}{c}\text { Interfaz usable y visualmente } \\
\text { Atractiva }\end{array}$ \\
\hline
\end{tabular}

La interactividad entre personas y máquinas nace bajo el concepto del comportamiento interactivo entre el aparato y la experimentación del humano, totalmente apartado del aspecto o apariencia visual de la máquina sus componentes y procesos internos o significados que transmitan (Fernández 2011). Las interfaces de usuario dependiendo de su nivel de interactividad pueden ser ubicadas en distintos grados, los cuales se tabularon en la Tabla 2.

Tabla 2: Grados de Interactividad

\begin{tabular}{c|c} 
Grado & Descripción \\
\hline Interactivo & $\begin{array}{c}\text { Comunicación entre sistema interfaz-humano } \\
\text { constante, respuesta mutua }\end{array}$ \\
Reactivo & $\begin{array}{c}\text { Comunicación entre interfaz-humano mínima, } \\
\text { respuesta unilateral }\end{array}$ \\
Estático & Comunicación nula entre interfaz-humano
\end{tabular}

El test de usuario basado en el tema de la interactividad se ejecutó en algunos colegios de la ciudad de Riobamba, según lo determinó la muestra calculada para tal propósito, para la aplicación del presente test fue indispensable la utilización de una aplicación controlada mediante una interfaz háptica, misma que fue estudiada con el fin de establecer grados de interactividad y similitud con la interfaz propuesta para el proyecto, dicha aplicación debía ser usada por el segmento de estudio sin ningún tipo de guía o ayuda de parte de los evaluadores, únicamente se debía intuir su manejo y configuración necesaria para alcanzar los mejores resultados.

Con estos antecedentes fue seleccionada una aplicación creada por Gameloft, cuya trayectoria se remonta al año 2011, existiendo ya varias actualizaciones y variaciones del video juego permitiendo a los usuarios jugarlo desde cualquier plataforma de dispositivo móvil. La totalidad de estudiantes de nivel secundario encuestados, considera que una pantalla táctil incrementa la interactividad dentro de una aplicación, de estos un $84 \%$ de usuarios considera que las aplicaciones actuales tienen un alto nivel de interactividad directa con el usuario. El nivel de Intervención del usuario dentro de la realización de tareas en una interfaz háptica se encuentra en un rango equilibrado siendo alrededor del $30 \%$ para nivel bajo, medio y alto; pero existe aún predominancia por el nivel más adecuado. Al momento de realizar las tareas, el usuario está en capacidad de formar parte de las actividades virtuales, mostrándose una igualdad del $36 \%$ entre el nivel más alto y el nivel más bajo de participación.

Sin duda las interfaces hápticas presentan muchas ventajas sobre otro tipo de interfaces gráficas existentes, sin embargo para usuarios con poco conocimiento de las mismas puede resultar complicado como lo señala Cabral cuando presenta los resultados de su estudio y manifiesta que sus experimentos muestran que el tiempo para la realización de tareas de apuntamiento simples es considerablemente más lento en comparación con un ratón y que su uso durante incluso breves períodos de tiempo causa fatiga (Cabral et al. 2005), sin embargo en la actualidad el dominio de las aplicaciones móviles y sus interfaces, demostró que fue una excelente opción el haber escogido una interfaz háptica táctil para el desarrollo del presente proyecto.

Los motores gráficos que permiten crear videojuegos para diferentes plataformas y que en este caso fueron considerados para crear los entornos gráficos $3 D$ son los siguientes:

- UDK de Epic Games (https://epicgames.com/)

- CryEngine de Crytek (http://www.crytek.com/)

- Unity 3D de Unity Technologies (https://unity3d.com/es/company)

Si bien cada uno de ellos presenta una serie de ventajas solo uno fue seleccionado para implementar las interfaces de la aplicación.

\section{Métodos y herramientas}

El motor gráfico seleccionado para el desarrollo del presente proyecto fue Unity 3D debido principalmente a los siguientes factores:

1. Posibilidad de disponer de una versión tanto para Windows como para Mac, aspecto importante considerando que los estudiantes que trabajaron en el diseño durante su carrera manejaron el entorno de desarrollo en Mac.

2. Además la versión de pago que es completa, brinda una versión gratuita con todas las facilidades técnicas y de diseño para cumplir con los objetivos planteados. 
3. Posibilidad de crear entornos 3D para PC, Mac y Web y lo más importante durante el desarrollo del proyecto estuvo habilitada la versión para realizar desarrollo para dispositivos iOS y Android, plataformas a las que está enfocado el proyecto.

4. Permite trabajar con iluminación global, efectos de post-procesado, light flare, vignetting, rayos de luz, profundidad de campo y corrección de color, entre otras.

5. Integración con la herramienta LabView.

6. Finalmente y quizá el más importante es que el equipo desarrollador durante su formación académica estudio Unity 3D para el desarrollo de videojuegos.

La selección de un entorno de desarrollo que permita conectar el hardware que controla la maqueta física de tren y las estaciones con la interfaz háptica 3D estuvo considerado entre las tres opciones que brinda la empresa National Instrument y que son:

- LabVIEW.

- LabWindows/CVI.

- Measurement Studio.

LabVIEW, software que proporciona un potente entorno de desarrollo gráfico fue seleccionado entre otros aspectos por:

- Integración de primera clase con hardware.

- Interfaces de usuario personalizadas.

- Extenso IP de análisis y procesamiento de señales.

- Sintaxis de programación gráfica

El proyecto Computational Thinking enabled by Robotmediated Interactions in a 3D Virtual Simulation of the Fukushima nuclear power plant (Vallance et al. 2015) fue tomado como ejemplo debido a que mostró un ambiente similar al que se buscaba alcanzar en el proyecto. Con las herramientas seleccionadas, el siguiente paso fue definir las actividades requeridas para realizar el simulador siguiendo las acciones generales de funcionamiento, el estudio realizado indica la manera correcta de elaborar un escenario para interacción usuario-máquina.

Bajo poligonaje. El modelado adecuado para una simulación fluida corresponde a un control en el número de polígonos creados por el modelador, entre más polígonos posee el objeto, mayor será la cantidad de información que el motor de video juegos procese, ralentizando la simulación y perdiendo la continuidad de la interacción del usuario.

Texturizado. Las texturas de los diferentes objetos modelados deberán ser colocadas de manera correcta, abarcando el total de la superficie visible, logrando una simulación realista. El tamaño de pixeles en las imágenes utilizadas para texturizar objetos, beben ser inferiores, con el fin de economizar espacio virtual en la simulación.

Tamaño de terreno. Se recomienda terrenos pequeños, dependiendo del uso requerido por el diseñador, para una mejor vinculación con el espacio virtual asimilada por el usuario.
Basado en los puntos anteriores se realizó el modelado 3D y en base a los resultados parciales empezar a construir la maqueta física, en este caso la estación del tren de Riobamba. En general los pasos del Modelado 3D fueron los siguientes.

1. Se diseñó el objeto $3 \mathrm{D}$ con unidades reales (Fig. 1).

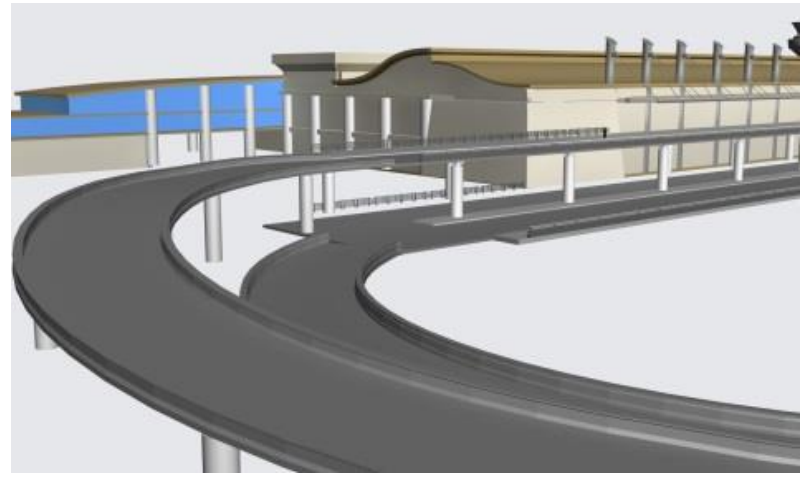

Figura 1: Modelado 3D.

2. Se construyó la maqueta de la estación de trenes (Fig.2).

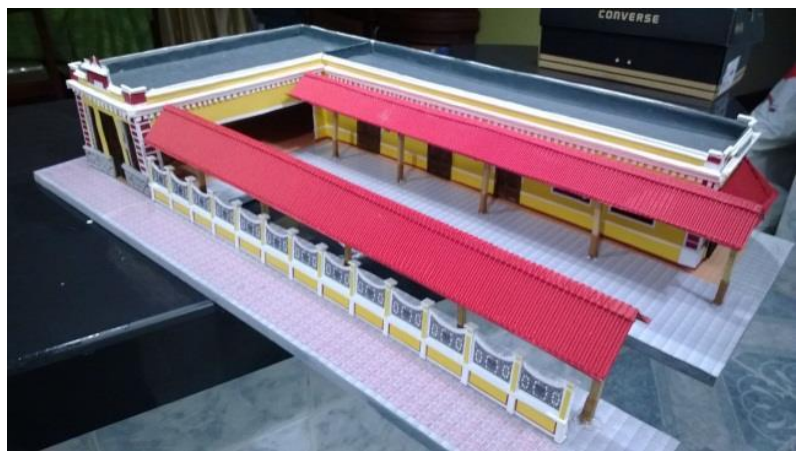

Figura 2: Maqueta de la Estación del tren.

3. Se diseñó en 3D el sistema de rieles y trenes y luego se construyó el modelo físico usando técnicas de ferro modelismo (Fig. 3).

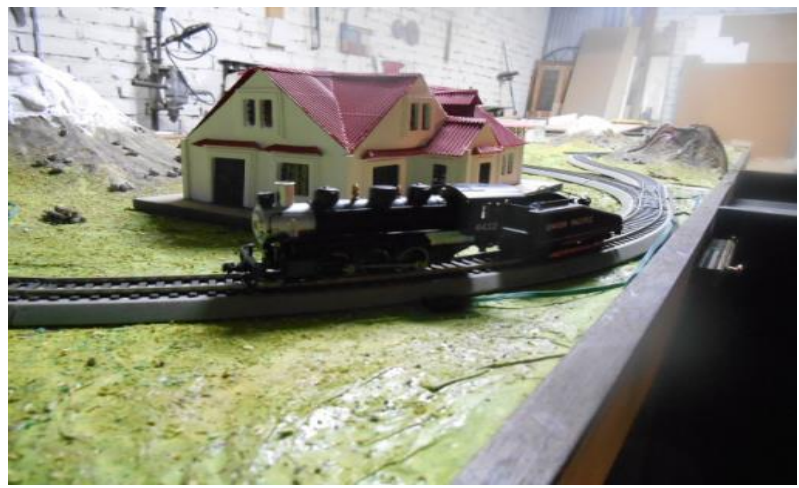

Figura 3: Sistema de trenes eléctricos.

4. Se integró en ambiente 3D, estación y trenes y se construyó la maqueta completa con las estaciones de trenes y el sistema de trenes eléctricos (Fig. 4).

5. Se construyeron componentes electrónicos para activar el sistema de modelos de trenes. 
6. Se diseñó una interfaz de comunicación entre la maqueta física y un programa instalado en un dispositivo móvil.

7. Al final se hicieron varias pruebas y se invitó a estudiantes para que realizaran la operación completa que consistía en "manejar" el tren de Riobamba, considerado Patrimonio Cultural, a través del dispositivo móvil en el que se instaló la aplicación.

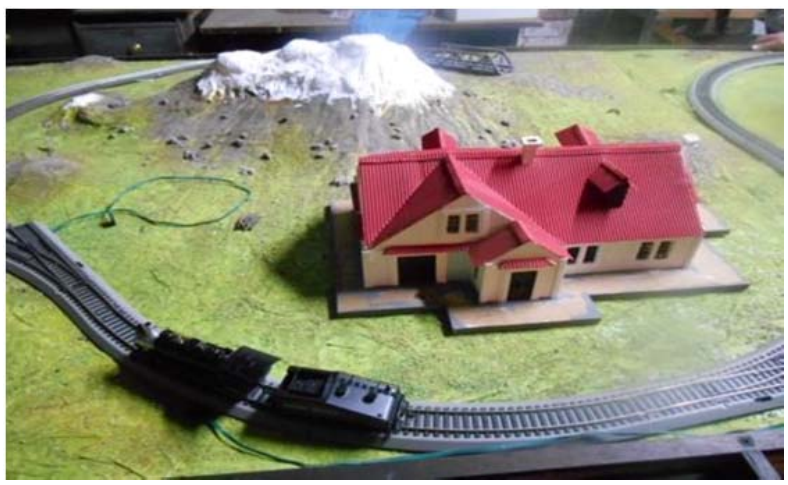

Figura 4: Sistema completo de maqueta y trenes.

La maqueta física fue activada mediante comandos realizados desde el celular. Se crearon varios controles, el control de movimiento determina el desplazamiento del personaje dentro del entorno debe ser fluido y controlado para lograr un desarrollo normal y realista de las actividades en el ambiente 3D. El control de eventos permite controlar luces, semáforos, sonidos del tren y el control de interacción permite al usuario interactuar con la maqueta física realizando las siguientes funciones:

- Movimiento del tren eléctrico mediante una interfaz similar a la del tren a vapor (Fig. 5).

- Activación de pitos.

- Activación de sonidos.

- Activación de letreros con información de la ciudad o sitio turístico (ej. Monte Chimborazo) donde el tren está pasando (Fig. 6).

- Información sobre rutas del tren

- Ingreso a la estación

- Efectos especiales en la maqueta como sonidos de la ciudad / campo.

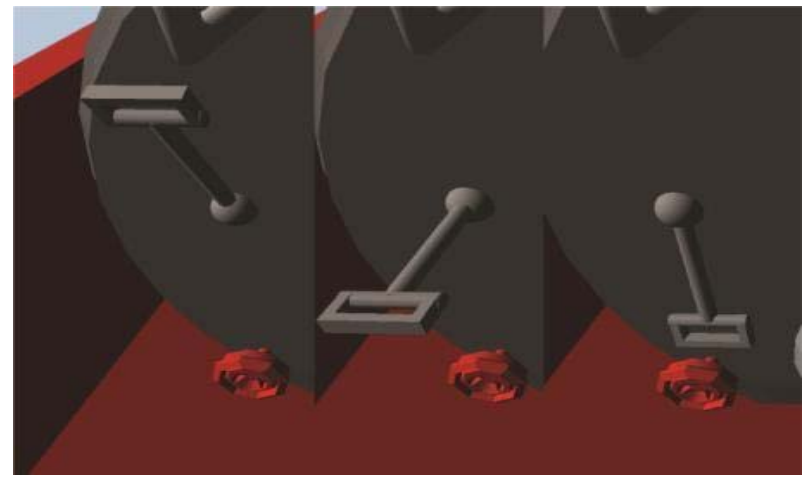

Figura 5: Interfaz que simula las palancas de una locomotora real.

Para la programación de los componentes hápticos y lógicos se utilizó el programa Unity 3D Personal Edition con sus opciones de motor de videojuegos. Para las conexiones físicas se diseñó a partir del software LabView.

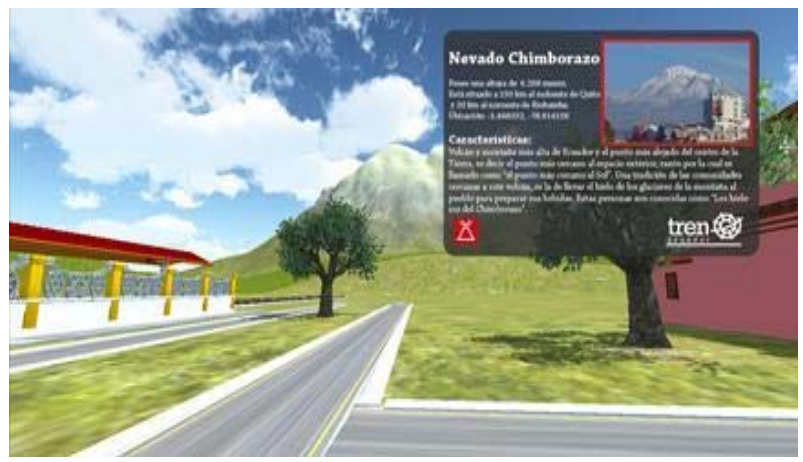

Figura 6: Información sobre el Chimborazo.

Con la información tanto de LabView y de Unity 3D se integró en una consola de control para manipular todos los eventos antes descritos (Fig. 7).

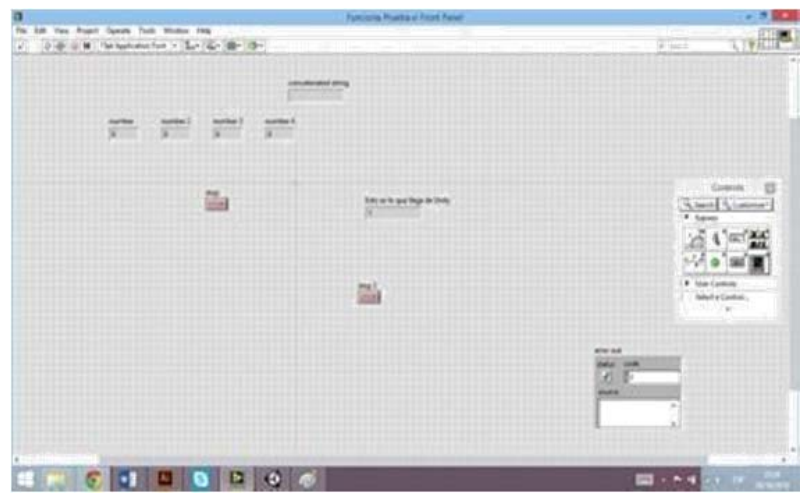

Figura 7: Consola datos UNITY-LabView-UNITY.

Todos los requerimientos y necesidades que se obtuvieron luego de la investigación realizada en este proyecto han sido implementados al momento de la creación de esta aplicación y su interfaz. Todos los componentes, requerimientos y la aplicación en general se los diseño siguiendo pautas de procesos de modelado digital (Vaughan 2012).

Se debe recalcar que, para lograr una vinculación virtual - real como la que se presenta en el presente sistema fue necesaria la utilización de dos aplicaciones que permitieron obtener los mejores resultados en cada uno de sus procesos, LabView producto más famoso de National Instruments encargado de controlar el sistema electrónico presente en la maqueta a través del uso de la tarjeta DAQ OEM 6009 de la misma empresa, esta recibe y emite las señales necesarias para el encendido, apagado y otros controles de los componentes electrónicos, como sensores QRD, luces leds, potenciómetros, etc.

Por otro lado Unity 3D, aplicación utilizada para programar y estructurar la interfaz de la aplicación que posteriormente permitirá tener un control táctil de los elementos inmersos dentro del mundo virtual. La conexión que se realiza entre estas dos aplicaciones es bidireccional, lo que quiere decir que cuando la aplicación configurada con Unity 3D reciba una indicación mediante el dispositivo háptico, enviará una señal a LabView y este ejecutará una secuencia. De 
igual modo, cuando LabView reciba una señal de los sensores ubicados en la maqueta real, enviará un dato a Unity 3D para que este lo interprete y ejecute una secuencia dentro del sistema virtual. Este tipo de conexión se la conoce como "Conexión por Sockets" y consiste en la apertura de clientes y servidores, que escuchen o envíen señales. Estos sockets funcionan en base a la dirección IP del ordenador, quien puede enviar o recibir señales desde cualquier dispositivo conectado a la red, pudiendo ser este un ordenador, un dispositivo móvil, o incluso el mismo ordenador principal, la conexión permite descartar la necesidad de un servicio de internet, necesitando únicamente una red WiFi o WLAN para funcionar correctamente.

\section{Resultados}

La operación del sistema fue realizado en varias etapas por estudiantes que previamente fueron seleccionados. Un número total de 20 fueron seleccionados por su conocimiento en el uso del celular de manera ampliada.

Luego de la tabulación de los resultados obtenidos se pudo determinar que el $95 \%$ de los estudiantes que colaboraron con este test, pudieron realizar las tareas de una manera fácil, el $100 \%$ opinaron que los mensajes mostrados por la aplicación fueron apropiados, el 95\% mostraron facilidad para desplazarse por el entorno, mientras el $90 \%$ de estudiantes evaluados consideran que la aplicación posee un nivel de usabilidad óptimo y un $10 \%$ lo considera Usable. En la segunda parte de la investigación, se obtuvieron resultados que muestran un $100 \%$ de interactividad de los estudiantes. Con los resultados obtenidos de la tabulación de las dos evaluaciones, se muestra un aumento notable del nivel de percepción de la usabilidad e interactividad, quedando así comprobada la hipótesis planteada.

\section{Conclusiones}

El uso de una interfaz háptica en una aplicación 3D, mejora la usabilidad e interactividad del usuario en el aprendizaje y manipulación de un sistema tanto virtual como físico. Un sistema virtual - real ayuda a mejorar la retención de información y permite al usuario participar activamente en entornos virtuales. La tecnología móvil con su versatilidad y prestaciones se constituyó en el dispositivo ideal para dar a conocer el funcionamiento e historia del tren de Riobamba y contribuir con la recuperación de uno de los patrimonios culturales de la ciudad. El uso de interfaces hápticas en la actualidad constituyen una necesidad frente a la constante evolución de los sistemas informáticos, la importancia de incrementar el grado de usabilidad e interactividad obliga a la actualización en la forma de controlar las aplicaciones y sus interfaces. La cantidad de usos y aplicaciones que poseen las interfaces hápticas, permiten la vinculación de estas en diferentes campos de acción, como la mecánica, la medicina, la astronomía, la educación, la industria, la cultura y demás campos que requieran la interacción hombremáquina.

\section{Agradecimientos}

Se agradece a la ESPOCH - FIE por la facilidad física brindada a Leonardo Arévalo, Miguel Ángel Urgilez, Martha Dávalos y Patricia Avalos por su valiosa participación en la programación de los sistemas y por la recolección de datos in situ.

\section{Referencias}

APARICI, R. y SILVA, M., 2012. Pedagogy of Interactivity/Pedagogía de la interactividad. Comunicar,19(38), 51. DOI: http://dx.doi.org/10.3916/C38-2012-02-05

CABRAL, M., MORIMOTO, C. y ZUFFO, M., 2005. On the usability of gesture interfaces in virtual reality environments. In Proceedings of the 2005 Latin American conference on Human-computer interaction (CLIHC '05). ACM, New York, NY, USA, pp. 100-108. DOI: http://dx.doi.org/10.1145/1111360.1111370

CARRASQUILLA, A., 2012. Teleoperación de un vehículo remoto en un medio de acceso inalámbrico mediante el uso de una interfaz háptica. Vol. 25, №. Extra 5, 2012 pp. 5-13.

ECURED, 2016. Tecnología háptica. Disponible: http://www.ecured.cu/Tecnología_háptica [5/30,2016].

FERNÁNDEZ, M., 2011. Modelado, texturizado y ajuste de malla. Postproducción digital. Madrid: Bubok, pp. 105-132.

HURTADO, L. y FORERO, J., 2014. Metodología de evaluación de usabilidad de interfaces humano-máquina. Revista Tecnura, 18, pp. 103-114. DOI: http://dx.doi.org/10.14483/udistrital.jour.tecnura.2014.SE1.a08

LÓPEZ, J., TORRES, J., GIMÉNEZ, A., GALIANA, I. y FERRE, M., 2010. Interfaz háptica para tareas de manipulación, XVIII Congreso Nacional de Ingeniería Mecánica.

NIELSEN, M., STÖRRING, M., MOESLUND, T., y GRANUM, E., 2003. A procedure for developing intuitive and ergonomic gesture interfaces for $\mathrm{HCl}$. In Gesture-Based Communication in Human-Computer Interaction, pp. 409420. Springer Berlin Heidelberg. DOI: 10.1007/978-3-540-24598-8_38

MÉNDEZ, R., CASTRO, G. y CASTRO, M., 2015. Un análisis de la adicción a los dispositivos móviles y su impacto en el rendimiento académico de los estudiantes de la licenciatura en informática administrativa del centro universitario UAEM TEMASCALTEPEC. Atlante. Cuadernos de Educación y Desarrollo.

VALLANCE, M., MARTIN, S. y NAAMANI, C., 2015. A situation that we had never imagined: post-Fukushima virtual collaborations for determining robot task metrics. International Journal of Learning Technology, Vol. 10, No. 1, pp. 30-49. DOI: http://dx.doi.org/10.1504/IJLT.2015.069453

VAUGHAN, W., 2012. Modelado Digital (Espacio de Diseño). Washington - USA., Anaya Multimedia. 